\title{
Multiple-Coated PLGA Nanoparticles Loading Triptolide Attenuate Injury of a Cellular Model of Alzheimer's Disease
}

\author{
Lu Jia, ${ }^{1,2}$ Xiao-qi Nie, ${ }^{2}$ Hong-ming Ji, ${ }^{2}$ Zhi-xiang Yuan $\mathbb{D}^{3}$ and Rong-shan Li $\mathbb{D}^{2}$ \\ ${ }^{1}$ Second Hospital of Shanxi Medical University, Taiyuan, Shanxi, China \\ ${ }^{2}$ Shanxi Provincial People's Hospital, Taiyuan, Shanxi, China \\ ${ }^{3}$ College of Pharmacy, Southwest Minzu University, Chengdu, Sichuan, China
}

Correspondence should be addressed to Rong-shan Li; rongshanli@126.com

Received 25 August 2020; Revised 11 January 2021; Accepted 20 January 2021; Published 25 February 2021

Academic Editor: Ali Nokhodchi

Copyright (c) $2021 \mathrm{Lu}$ Jia et al. This is an open access article distributed under the Creative Commons Attribution License, which permits unrestricted use, distribution, and reproduction in any medium, provided the original work is properly cited.

\begin{abstract}
Alzheimer's disease (AD) is the most common neurodegenerative disease, which is associated with extracellular deposition of amyloid- $\beta$ proteins $(\mathrm{A} \beta)$. It has been reported that triptolide (TP), an immunosuppressive and anti-inflammatory agent extracted from a Chinese herb Tripterygium wilfordii, shows potential neuroprotective effects pertinent to AD. However, the clinical use of $\mathrm{TP}$ for $\mathrm{AD}$ could be hampered due to its high toxicity, instability, poor water solubility, and nonspecific biodistribution after administration. In this paper, we reported a kind of multiple-coated PLGA nanoparticle with the entrapment of TP and surface coated by chitosan hydrochloride, Tween-80, PEG20000, and borneol/mentholum eutectic mixture (MC-PLGA-TP-NP) as a novel nasal brain targeting preparation for the first time. The obtained MC-PLGA-TP-NP was $147.5 \pm 20.7 \mathrm{~nm}$ with PDI of $0.263 \pm 0.075$, zeta potential of $14.62 \pm 2.47 \mathrm{mV}$, and the entrapment efficiency and loading efficiency of $93.14 \% \pm 4.75 \%$ and $1.17 \pm 0.08 \%$, respectively. In comparison of TP, MC-PLGA-TP-NP showed sustained-release profile and better transcellular permeability to Caco-2 cells in vitro. In addition, our data showed that MC-PLGA-TP-NP remarkably reduced the cytotoxicity, attenuated the oxidative stress, and inhibited the increase of the intracellular $\mathrm{Ca}^{2+}$ influx in differentiated PC12 cells induced by $\mathrm{A} \beta_{1-42}$. Therefore, it can be concluded that MC-PLGA-TP-NP is a promising preparation of TP, which exerts a better neuroprotective activity in the AD cellular model.
\end{abstract}

\section{Introduction}

Alzheimer's disease (AD), the most common neurodegenerative disease, is a major form of dementia characterized clinically by progressive cognitive impairments, including memory, judgment, decision-making, and language $[1,2]$. However, the pathogenesis and etiology of $\mathrm{AD}$ are still far from well understanding. The pathological changes observed in brains of $\mathrm{AD}$ patients reflect a series of chronic inflammatory processes, in which response is characterized by the presence of abundant activated microglia. Moreover, mounting evidence indicates that reactive astrocyte proliferation is associated with extracellular deposition of amyloid- $\beta$ proteins $(A \beta)$, formation of neurofibrillary tangles and amyloid plaques, and increased expression of proinflammatory cytokines and complement components, which in turn induce neuroinflammation in the brain $[3,4]$. Therefore, more and more studies have focused on the roles and mechanisms of anti-inflammatory and immune modulatory agents for $\mathrm{AD}$ treatment.

Triptolide (TP), a major bioactive ingredient extracted from the roots, leaves, flowers, and fruits of Tripterygium wilfordii widely used as a herbal medicine [5], has been shown to possess multiple pharmacological functions $[6,7]$, including potential neuroprotective effects pertinent to $\mathrm{AD}$ [8]. However, the clinical use of TP is hampered due to its high toxicity, instability, poor water solubility, and nonspecific biodistribution after administration. Additionally, the major hurdle for delivering drugs to brain is the presence of the blood-brain barrier (BBB) which is the complicated network of blood vessels having tightly packed endothelial cells for the separation of the brain from the blood circulatory system [9]. 
Thus, in order to realize the efficient therapy of TP for AD, it is necessary to develop novel TP preparation for overcoming the mentioned limitation.

With the rapid development of nanotechnology, biodegradable polymers have been extensively applied in pharmaceutical and biomedical formulations during the past few decades [10]. Especially, poly(D,L-lactic-co-glycolic acid) (PLGA) approved by the Food and Drug Administration (FDA) has been successfully applied for the formation of nanoparticles in lab and in clinic because of good biocompatibility and biodegradability [11]. The entrapment of TP into PLGA nanoparticles is considered a smart way to increase the stability and water solubility, decrease the toxicity, and enhance the specific distribution of TP after administration [12]. Intranasal administration could noninvasively realize drug-loaded nanoparticles to bypass the BBB to deliver therapy agents for the treatment of brain disease by the olfactory pathway which is a reliable alternative to achieve desire therapeutic effects at lower doses while minimizing side effects [9, 13]. Therefore, the development of TP-loaded PLGA nanoparticles is an ideal method to improve the treatment for AD.

In previous study, we have developed a novel delivery system to promote targeting profile and reduce toxicity of TP [14]. In our current work, we focus on bypassing the $\mathrm{BBB}$ using intranasal administration of PLGA nanoparticles coated by chitosan hydrochloride, Tween-80, PEG20000, and borneol/mentholum eutectic mixture. TP was also encapsulated into the multiple-coated PLGA nanoparticles as a novel brain targeting preparation (MC-PLGA-TP-NP) for nasal drug delivery with the aim of overcoming the clinical limitation. Then, MC-PLGA-TP-NP was characterized, and its cumulative release was evaluated. To prove the better permeation to the olfactory neurons, we performed the transcellular permeability study on MC-PLGA-TP-NP through Caco-2 monolayer cells. In addition, a cellular model of AD (the differentiated PC12 cells) induced by $\mathrm{A} \beta_{1-42}$ was established to evaluate the attenuation of cytotoxicity, reactive oxygen species (ROS) generation, and calcium influx after the treatment of MC-PLGA-TP-NP using free TP as a contrast.

\section{Materials and Methods}

2.1. Chemicals and Reagents. Triptolide (TP, purity >99\%), PLGA (with a copolymer ratio of lactide:glycolide 50:50 and $\mathrm{Mw}: 40-75 \mathrm{kDa})$, poloxamer 188 (F-68, Mw:8350 Da), amyloid beta-peptide ${ }_{1-42}\left(\mathrm{~A} \beta_{1-42}\right)$, and 3-(4,5-dimethylthiazol-2-yl)-2,5-diphenyltetrazolium bromide (MTT) were supplied by Sigma-Aldrich Co. Ltd. (Gillingham, UK). Dichlorodihydrofluorescein diacetate (DCFH-DA), fluo-3 acetoxymethyl ester (fluo-3-AM), LysoTracker Red, and $4^{\prime}$ ,6-diamidino-2-phenylindole (DAPI) were from Molecular Probes (Eugene, OR, USA). Fetal bovine serum (FBS), horse serum (HS), nerve growth factor (NGF), penicillin, and streptomycin were provided by GIBCO, Invitrogen Corporation (Carlsbad, CA, USA). Chitosan hydrochloride (degree of deacetylation, $80 \% \sim 90 \%$, viscosity, $30 \mathrm{mPa} . \mathrm{s}$ ) was obtained from Shanghai KABO Trading Company (Shanghai, China). Polyethylene Glycol (PEG, MW = $20 \mathrm{kDa}$ ), borneol, mentho- lum, and Tween-80 were purchased from Chengdu Kelong Chemical Company (Chengdu, China). The other chemicals were of analytical reagent grade.

2.2. Preparation of MC-PLGA-TP-NP. MC-PLGA-TP-NP was prepared according to our previous method with minor modification [13]. In brief, $415 \mathrm{mg}$ PLGA, $5 \mathrm{mg} \mathrm{TP}$, and $150 \mathrm{mg}$ borneol/mentholum eutectic mixture $(1: 3$, w/w) were dissolved into $50 \mathrm{ml}$ of ethanol/acetone $(3: 2)$ followed by a dropwise addition to $50 \mathrm{ml}$ of $1 \% \mathrm{w} / \mathrm{v}$ poloxamer 188 solution containing $250 \mathrm{mg}$ PEG with continuous stirring on a magnetic stirrer at room temperature to obtain a dispersion. After vacuum rotary evaporation to remove the organic solvent, $250 \mathrm{mg}$ Tween- 80 and $50 \mathrm{mg}$ chitosan hydrochloride were added to the dispersion while being stirred magnetically for $2 \mathrm{~h}$. Then, the dispersion was centrifuged at $25000 \mathrm{rpm}$ for $30 \mathrm{~min}$. The supernatant was collected for HPLC analysis while the precipitate was obtained, namely, MC-PLGA-TP$\mathrm{NP}$, which was dispersed into $10 \mathrm{ml}$ ultrapure water containing $0.5 \mathrm{~g}$ of lactose as lyoprotectant. The obtained dispersion was prefrozen using ultra-low temperature refrigerator at $-80^{\circ} \mathrm{C}$ for $24 \mathrm{~h}$ followed by lyophilization in freeze-dryer equipment (LyoQuest, Telstar, Spain) under $0.4 \mathrm{mbar}$ vacuums for $24 \mathrm{~h}$ and a condenser temperature of $-40^{\circ} \mathrm{C}$ to form a powder for storage at $4^{\circ} \mathrm{C}$.

2.3. Entrapment Efficiency and Loading Efficiency. HPLC (Aglient 1260, Aglient Technologies, Santa Clara, CA) was equipped with an online degasser, a quaternary pump, an autosampler, a column thermostat, and an Aglient C18 $(250 \mathrm{~mm} \times 4.6 \mathrm{~mm}, 5 \mu \mathrm{m})$ analytical column for chromatographic separation. All separations were executed at $30^{\circ} \mathrm{C}$ with a mobile phase of acetonitrile/water (25:75), a flow rate of $1.0 \mathrm{ml} / \mathrm{min}$, and a $10 \mu \mathrm{L}$ sample volume injected into the HPLC system for analysis. The UV detector was operated at $218 \mathrm{~nm}[14,15]$. Peak identification was carried out by comparing the retention times of the samples with those of reference standards. The centrifugation method was involved to calculate the entrapment efficiency and loading efficiency. The collected supernatant from the dispersion after the centrifugation at $25000 \mathrm{rpm}$ was diluted appropriately and then injected into HPLC to assess the amount of TP in the supernatant. Entrapment efficiency (EE) and loading efficiency (LE) were determined according to the following equation [16].

$\mathrm{EE} \%=($ total amount of $\quad \mathrm{TP}-$ the amount of $\mathrm{TP}$ in the supernatant)/total amount of TP $\times 100 \%$.

$\mathrm{LE} \%=\quad$ (total amount of TP - the amount of TP in the supernatant $) /[$ (total amount of TP - the amount of TP in the supernatant $)+$ total amount of PLGA] $\times 100 \%$.

2.4. Characterization of MC-PLGA-TP-NP. MC-PLGA-TPNP freeze-dried powder was appropriately dispersed with distilled water. The particle size and zeta potential of MCPLGA-TP-NP were determined by Malvern Zetasizer Nano ZS90 (Malvern Instruments Ltd, Malver, UK). S4800 Transmission electron microscope (Hitachi Ltd, Tokyo, Japan) was applied to observe the features of MC-PLGA-TP-NP. Briefly, MC-PLGA-TP-NP suspension was dripped onto a dedicated 


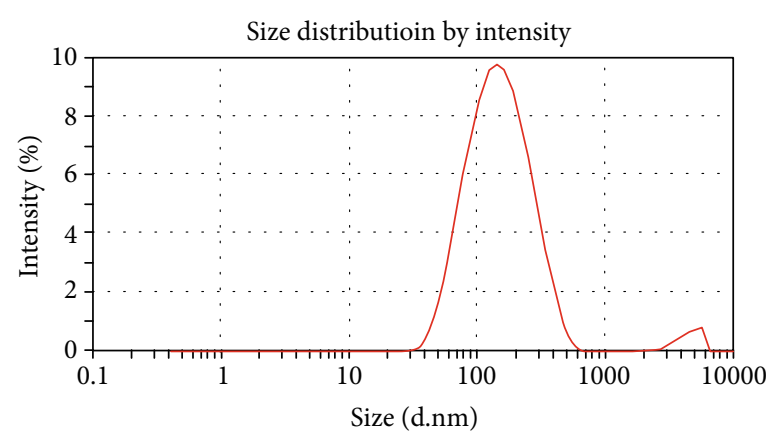

(a)

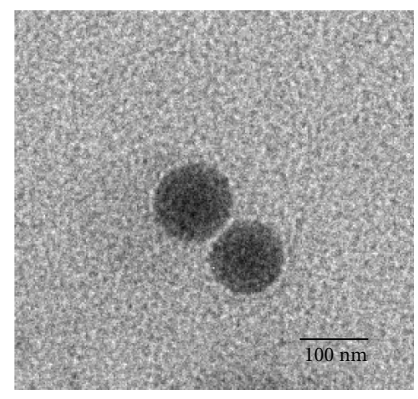

(c)

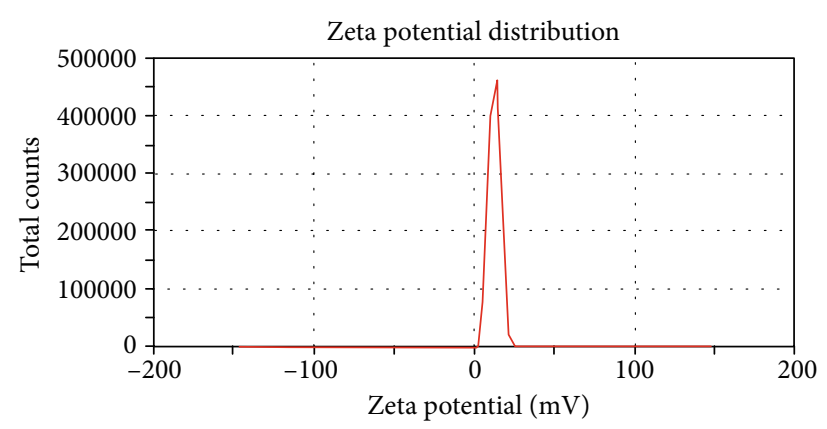

(b)

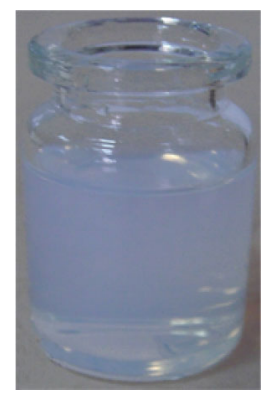

(d)

FIGURE 1: Size distribution (a), zeta potential distribution (b), and TEM image (c) of MC-PLGA-TP-NP redispered into deionized water (d).

copper mesh and then negatively stained with $4 \%$ phosphotungstic acid solution for $30 \mathrm{~s}$. After drying at ambient temperature, the nanoparticles were observed under the transmission electron microscope.

2.5. In Vitro Release. In vitro release studies were carried out in $0.05 \mathrm{M}$ phosphate buffer solutions (PBS, containing $0.1 \%$ Tween 80) at $\mathrm{pH} 4.0$ and $\mathrm{pH} 7.4$ using dynamic membrane dialysis. $5 \mathrm{ml}$ of MC-PLGA-TP-NP or TP suspension (with an equivalent dose of $5 \mathrm{mg} \mathrm{TP}$ ) was added in a dialysis bag (3500 Da) and then dialyzed against $95 \mathrm{ml}$ PBS in $250 \mathrm{ml}$ beaker situated in oscillating water bath at a temperature of 37 $\pm 0.5^{\circ} \mathrm{C}$. Samples $(1 \mathrm{ml})$ were obtained from the release medium at arranged time points (5, $30 \mathrm{~min}, 1,2,4,8,12$, and $24 \mathrm{~h}$ ) for determining the amount of TP that diffused through the dialysis bag. Meanwhile, $1 \mathrm{ml}$ of fresh release medium preheated at $37^{\circ} \mathrm{C}$ was added to maintain a constant volume at the above prearranged time points. Samples were analyzed by the HPLC method as described previously. Cumulative release characteristics of MC-PLGA-TP-NP or TP suspension were described and compared.

2.6. Cell Culture. The human epithelial cell line (Caco-2) purchased from American Type Culture Collection (Rockville, Maryland) was cultured in DMEM medium containing $10 \%$ FBS, $2 \mathrm{mM}$ glutamine, $100 \mu \mathrm{g} / \mathrm{ml}$ streptomycin, and $100 \mathrm{U} / \mathrm{ml}$ penicillin and maintained in a humidified atmosphere containing $5 \% \mathrm{CO}_{2}$ at $37^{\circ} \mathrm{C}$. Rat pheochromocytoma (PC12) cells kindly provided by Chinese Academy of Sciences were grown in RPMI1640 supplemented with 5\% FBS, $10 \% \mathrm{HS}, 100 \mu \mathrm{g} / \mathrm{ml}$ streptomycin, and $100 \mathrm{U} / \mathrm{ml}$ penicillin and incubated in a humidified atmosphere containing 5\% $\mathrm{CO}_{2}$ at $37^{\circ} \mathrm{C}$. Then, PC12 cells were plated onto 6-well cul- ture plates or 96-well culture plates coated with poly-Dlysine at a density of $1 \times 10^{5}$, and the medium containing $2.5 \mathrm{ng} / \mathrm{ml} \mathrm{NGF}$ was replaced 2 3 times for a week to obtain neuronal differentiated PC12 cells. All subsequent experiments in the present study were performed with the differentiated PC12 cells.

2.7. Transcellular Permeability. Caco-2 cells were seeded on the apical chamber of 6-well Transwell ${ }^{\mathrm{TM}}$ plates (Corning Inc., Corning, New York) at a density of $1.0 \times 10^{5} \mathrm{cell} / \mathrm{s} / \mathrm{cm}^{2}$. The DMEM-based culture medium was replaced with fresh medium every two days for about three weeks until they achieved a constant transepithelial electrical resistance (TEER, $>500 \Omega \cdot \mathrm{cm}^{2}$ ), indicating the formation of tight junctions in the monolayer. The apical (top) and basal (bottom) chambers were separated by a collagen-coated polytetrafluoroethylene membrane (4.52 $\mathrm{scm}^{2}$ surface, $24 \mathrm{~mm}$ diameter) with a pore size of $0.4 \mu \mathrm{m}$. Before the initiation of the experiments, the culture medium was removed, and the cell membranes were allowed to equilibrate in Hank's Balanced Salt Solution (HBBS, transport buffer) as follows: HBSS buffered with $10 \mathrm{mM}$ MES ( $\mathrm{pH} 6.0$ ) or $10 \mathrm{mM}$ HEPES (pH 7.4) was added to the apical $(1.5 \mathrm{ml})$ or basal chamber $(2.5 \mathrm{ml})$, respectively. After preincubation with transport buffer for $30 \mathrm{~min}$, permeability experiments were initiated by the addition of TP solution or MC-PLGA-TP-NP to the apical chamber at a final TP concentration of $20 \mu \mathrm{g} /$ well in HBBS. At prearranged time intervals $(15,30,60$, and $120 \mathrm{~min})$, an aliquot of $200 \mu \mathrm{l}$ sample was collected from the basal chamber and replaced by $200 \mu \mathrm{l}$ of a prewarmed HBSS buffer to maintain the total volume. Samples were analyzed by the HPLC method as described previously. Each experiment was repeated five times $(n=5)$. The cumulative permeability 
curves versus time of TP were plotted, and the apparent permeability coefficients $\left(P_{\text {app }}\right)$ were calculated using the equation as follows [17]:

$$
P_{a p p}=(\Delta Q / \Delta t) \times\left[1 /\left(A \times C_{0}\right)\right]
$$

$\Delta Q / \Delta t(\mu \mathrm{mol} / \mathrm{s})$ is the rate at which TP appears in the basal chamber, $C_{0}(\mu \mathrm{mol} / \mathrm{L})$ means the initial concentration of TP in the apical chamber, and $A\left(\mathrm{~cm}^{2}\right)$ represents the surface area of the cell monolayer.

2.8. Effects of TP or MC-PLGA-TP-NP on A $\beta_{1-42}$-Induced Cytotoxicity in PC12 Cells. According to previous study from Wang et al., $5 \mu \mathrm{M} \mathrm{A} \beta_{1-42}$ as a final concentration was incubated with the differentiated PC12 cells to induce the cytotoxicity for evaluating the protective effects of TP and MCPLGA-TP-NP [18]. Different concentrations of TP solution $(0 \mathrm{ng} / \mathrm{ml}, 0.1 \mathrm{ng} / \mathrm{ml}, 0.5 \mathrm{ng} / \mathrm{ml}, 1.0 \mathrm{ng} / \mathrm{ml}, 5.0 \mathrm{ng} / \mathrm{ml}$, and $10 \mathrm{ng} / \mathrm{ml}$ ) and MC-PLGA-TP-NP (with an equivalent dose of TP solution) were added to the differentiated PC12 cells, respectively. The medium containing $1 \%$ DMSO was served as control. After the incubation for $24 \mathrm{~h}$, the cell viability was determined by the MTT assay. Each experiment was repeated five times $(n=5)$.

2.9. Measurement of ROS Generation. The production of intracellular ROS induced by $\mathrm{A} \beta_{1-42}$ under different conditions was measured by dichlorodihydrofluorescein diacetate (DCFH-DA), a membrane-permeable probe. The differentiated PC12 cells were exposed to $5 \mu \mathrm{M} \mathrm{A} \beta_{1-42}$, and then TP solution or MC-PLGA-TP-NP (with an equivalent dose of TP solution) was added to provide $5.0 \mathrm{ng} / \mathrm{ml}$ of TP concentration for $24 \mathrm{~h}$, respectively. The cells exposed to the medium only were served as control. After incubation with $10 \mu \mathrm{M}$ DCFH-DA at $37^{\circ} \mathrm{C}$ for $1 \mathrm{~h}$, cells were washed with PBS for three times. The methodology was performed according to the procedures described in the ROS assay kit, and all procedures were carried out in the dark. The intracellular accumulation of ROS was immediately analyzed by a CytomicsFC 500 flow cytometry (Beckman Coulter, USA). 10,000 events were collected, and the level of ROS generation was calculated as follows: the level of $\operatorname{ROS}(\%)=$ the percentage of DCF - positive cells in the M1 region [19]. Each experiment was repeated five times $(n=5)$.

2.10. Measurement of Calcium Influx. Changes in intracellular calcium concentrations were monitored using Fluo-3$\mathrm{AM}$, a probe for $\mathrm{Ca}^{2+}$ measurement. Similarly, the differentiated cells seeded in 6-well plate were exposed to $5 \mu \mathrm{M} \mathrm{A} \beta_{1-42}$, and then TP solution or MC-PLGA-TP-NP was added to provide $5.0 \mathrm{ng} / \mathrm{ml}$ of TP concentration, respectively. The cells exposed to the medium only were served as control. After incubation for $48 \mathrm{~h}, 3 \mu \mathrm{M}$ Fluo-3-AM dissolved in HEPES solution (5.5 mM HEPES, $140 \mathrm{mM} \mathrm{NaCl}, 5 \mathrm{mM} \mathrm{KCl}, 1 \mathrm{mM}$ $\mathrm{CaCl}_{2}, 0.5 \mathrm{mM} \mathrm{MgCl}_{2}, 10 \mathrm{mM}$ glucose, 1\% DMSO, pH 7.4) was added into the chamber for another incubation at $37^{\circ} \mathrm{C}$ for $40 \mathrm{~min}$. Then, the cellular lysosomes and nucleus were stained with LysoTracker Red $(50 \mathrm{nM})$ and DAPI $(1 \mathrm{mg} / \mathrm{ml})$ at room temperature for $30 \mathrm{~min}$, respectively. Finally, the

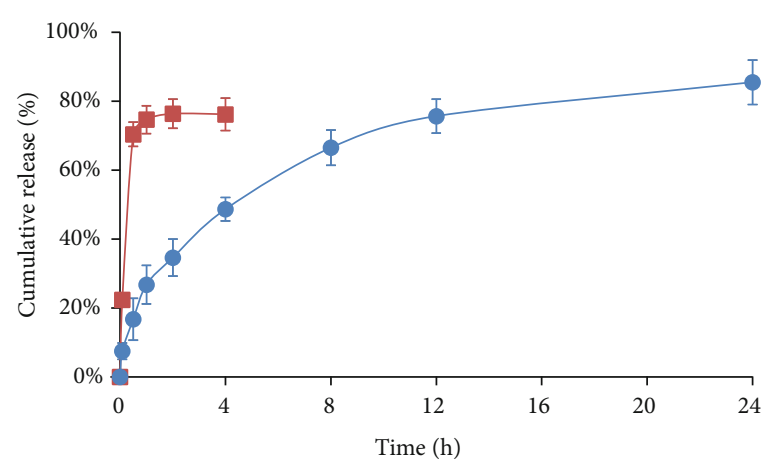

FIgURE 2: In vitro release profile of TP suspension ( $\square$ ) or MCPLGA-TP-NP ( $)$ in PBS $(\mathrm{pH}=7.4)$ using the dialysis bag diffusion technique. The experiment was repeated in triplicate, and data were given as the mean \pm standard deviation $(n=3)$.

cells were rinsed with HEPES solution for three times prior to the observation under Axio observer D1 fluorescence microscope (Carl Zeiss, Germany) with the excitation at $405 \mathrm{~nm}$ and $488 \mathrm{~nm}$ using a green channel (523 nm barrier filter), a red channel ( $630 \mathrm{~nm}$ barrier filter), and a blue channel (480 nm barrier filter). The measurement of fluorescence data (Fluo-3-AM) from individual cells was carried out using the MetaMorph software (Version 7.1, Universal imaging, USA).

2.11. Data Analysis. Data were compared with the SPSS 15.0 statistical package. Multiple comparisons of mean values were performed by one-way ANOVA with Fisher's least significant difference (LSD) test applied for posthoc comparisons at $95 \%$ confidence interval. $p<0.05$ was considered statistically significant.

\section{Results and Discussion}

It is reported that TP as a herb-derived drug shows a broad spectrum of biological profiles including anti-inflammatory, immunosuppressive, antifertility, antitumor activities, and neurotrophic and neuroprotective effects [7]. Nevertheless, the clinical use of TP is often discouraged due to its high toxicity, poor water solubility, and nonspecific biodistribution. Thus, how to enhance its solubility and improve targeting profile are key issues to be solved by developing new preparations. Various nanoparticles as useful vehicles were widely designed to load TP for the improvement of its shortcomings, including polylactic acid nanoparticle [20], solid lipid nanoparticle [21], and lipid-polymer hybrid nanoparticle [22]. In the study, a kind of multiple-coated PLGA nanoparticle by chitosan hydrochloride, Tween-80, PEG20000, and borneol/mentholum eutectic mixture was fabricated to load TP with the aims of enhancing the permeability of TP through $\mathrm{BBB}$ and exerting strong neuroprotective effects for the first time. As described in our previous study, free drugs can directly through the olfactory region to reach the cerebrospinal fluid or even the brain after intranasal administration. However, the most amount of drug will be suffered from adsorption and degradation under the action of the nasal enzymes and nasal mucosa, causing a very low amount of drug in the brain 


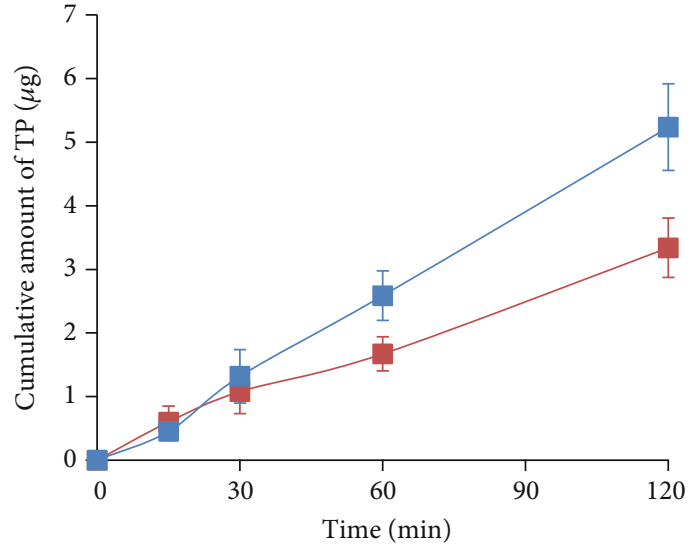

(a)

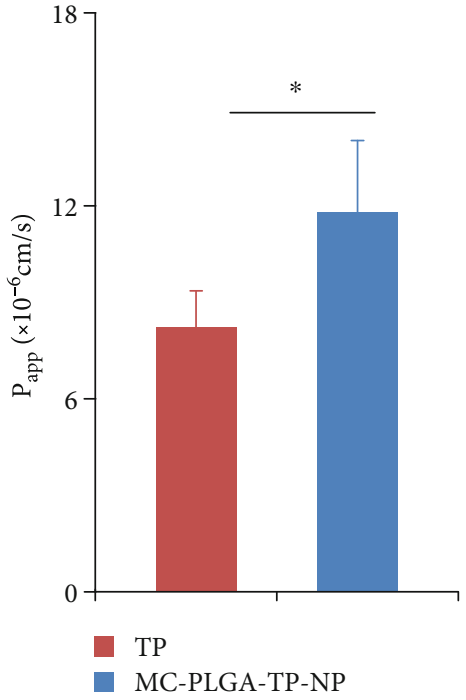

(b)

Figure 3: Transcellular permeability study on MC-PLGA-TP-NP through Caco-2 cells. (a) The time profiles of TP or MC-PLGA-TP-NP permeation through the Caco-2 monolayer seeded on Transwell plates; (b) The comparison of the calculated $P_{\text {app }}$ of TP and MC-PLGATP-NP. The experiment was repeated five times, and data were represented as the mean \pm standard deviation $(n=5)$. ${ }^{*}$ Significant difference $(p<0.05$, versus TP).

[13]. In order to increase the concentration of TP in the brain by nose-to-brain delivery, PLGA nanoparticles were used to protect TP from degradation and sustain release of TP while the coating of Tween- 80 and PEG on the surface of nanoparticles could avoid the clearance by macrophage and inhibit Pglycoprotein in the brain capillary endothelial cell membrane for the drug efflux function. Besides, the addition of chitosan hydrochloride and borneol/mentholum eutectic mixture is an effective way to modulate the permeability of BBB to facilitate the entry of therapeutic drugs into the brain.

HPLC analysis results showed that the retention time of TP was approximately $17.0 \mathrm{~min}$. The calibration curves of TP $\left(A=37857 \mathrm{C}+13127, R^{2}=0.9997 . A\right.$ and $C$ represented peak area and concentration, respectively), linear over the range of $2.16-54.0 \mu \mathrm{g} / \mathrm{ml}$, were used to calculate the concentration of TP in the supernatant from the dispersion. The methodology recoveries were among 95 103\% for all the three concentrations $(10,20,40 \mu \mathrm{g} / \mathrm{ml})$. Additionally, intraday and interday precision of TP was determined to be $1.74 \%$ and $2.64 \%$, respectively. Using the EE and LE as indexes determined by HPLC, the preparation process and formulation screening were performed according to our preliminary experiments (data not shown). After lyophilization in freeze dryer for $24 \mathrm{~h}$, the optimal MC-PLGA-TP-NP was obtained with the average EE of $93.14 \% \pm 4.75 \%$ as well as the average $\mathrm{LE}$ of $1.17 \pm 0.08 \%$.

The obtained MC-PLGA-TP-NP was well redispersed into deionized water (a sky-blue colored colloidal solution, Figure 1(d)) for the measurement of the particle size and zeta potential. As shown in Figures 1(a) and 1(b), the average particle size of the MC-PLGA-TP-NP was $147.5 \pm 20.7 \mathrm{~nm}$ with PDI of $0.263 \pm 0.075$ and zeta potential of $14.62 \pm 2.47 \mathrm{mV}$. As indicated in Figure 1(c), MC-PLGA-TP-NP was approxi- mately spherical with a certain uniform size distribution. The surface morphology was found to be relatively smooth, and no surface adsorbed particles were observed. There was a small peak appeared at about $4 \mu \mathrm{m}$ in the particle-size distribution graph (Figure 1(a)), which might be caused by the redispersion after lyophilization.

In vitro release behavior of TP suspension or MC-PLGATP-NP in PBS ( $\mathrm{pH}=7.4)$ was evaluated by dynamic membrane dialysis [23]. As shown in Figure 2, a rapid release within 0.5 h (about 70\%) was observed for TP suspension and then reached a plateau within $2 \mathrm{~h}$ (about $80 \%$ ). The release rate of TP from MC-PLGA-TP-NP was also evaluated. The first phase was a $26.75 \%$ release within the first $1 \mathrm{~h}$ mainly because TP adsorbed on the surface of nanoparticles could quickly diffuse in the PBS initially. And then a slow release, the second phase, was afterward for a period of $24 \mathrm{~h}$, which may be caused by TP diffusion from PLGA matrix. The maximum release rate of TP from MC-PLGA-TP-NP was more than $80 \%$ within $24 \mathrm{~h}$, indicating sustainedrelease profile of nanoparticles, which might be helpful for promoting the efficacy of neurotherapeutics. Notably, the cumulative release rate for both $\mathrm{TP}$ suspension and $\mathrm{MC}$ PLGA-TP-NP in PBS were much less than 100\%, maybe because of the degradation of TP in the release medium.

Deep administration of drugs to the nasal cavity approached nasal mucosa, leading to direct transmission of drug into the brain via the olfactory pathway, which consists of olfactory neurons with ability to carry drugs from olfactory mucosa to the brain [9]. As reported, nanoparticles, as excellent platforms for direct delivery of drug to the brain after intranasal administration by protecting drug from biological and chemical degradation and inhibiting the efflux of P-glycoprotein, were designed to encapsulate and deliver various 

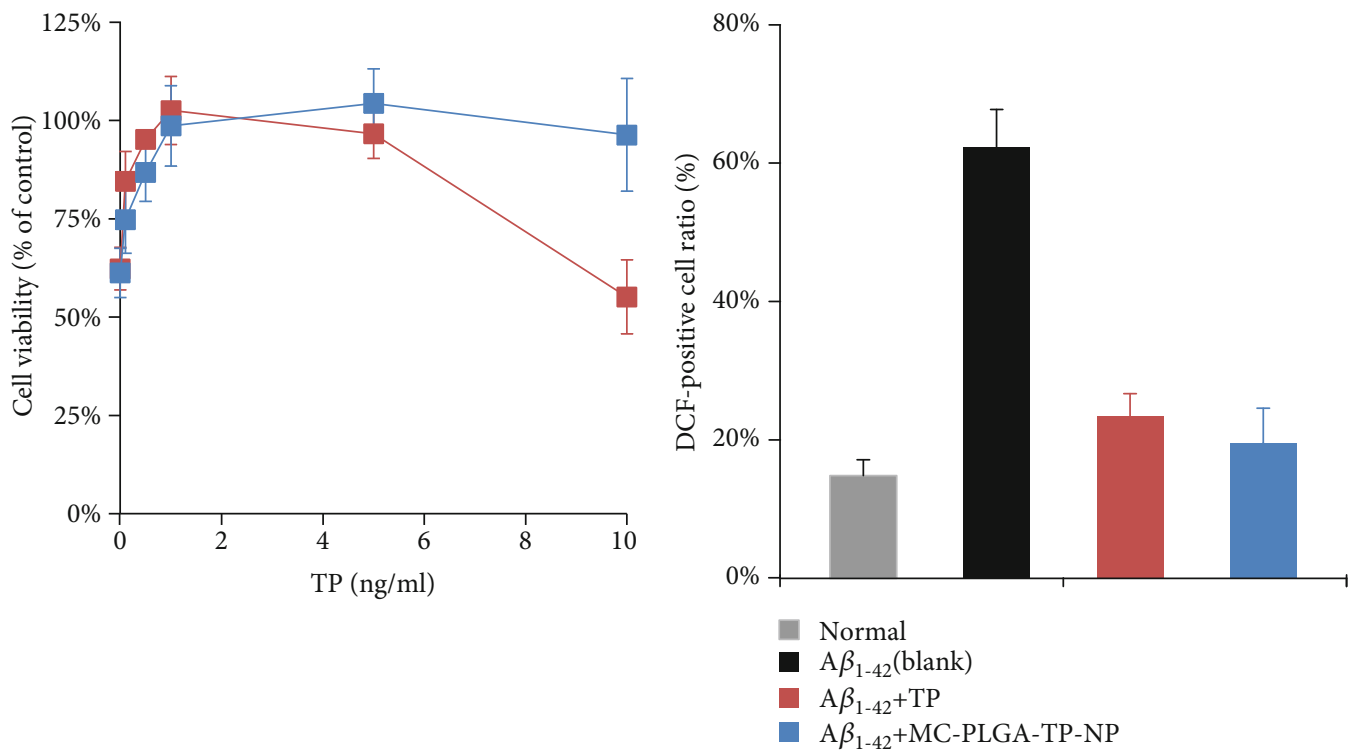

(a)

(b)

FIGURE 4: The attenuation of cytotoxicity (a) and ROS generation (b) in the differentiated PC12 cells induced by A $\beta_{1-42}$ after the incubation with TP or MC-PLGA-TP-NP for $24 \mathrm{~h}$. The experiment was repeated five times, and data were represented as the mean \pm standard deviation $(n=5)$.

therapeutic agents across the olfactory pathway to the brain [24-26]. It is unknown if the designed MC-PLGA-TP-NP was involved in the intracellular transport. We assumed that it could be based on literature mentioned above. Herein, we performed the transcellular permeability study on MCPLGA-TP-NP through Caco-2 cells. Figures 3(a) and 3(b) shows the time profiles and the calculated $P_{\text {app }}$ of TP or MC-PLGA-TP-NP permeation through the Caco-2 monolayer seeded on Transwell plates. The results demonstrated that free TP molecules exhibited better transcellular permeability than MC-PLGA-TP-NP at the beginning, whereas the amount of MC-PLGA-TP-NP increased sharply after incubation for $30 \mathrm{~min}$. The level of determined TP in the basal chamber of the MC-PLGA-TP-NP group was 1.5-fold higher than that of the TP group at 120 min (Figure 3(a)). The $P_{\text {app }}$ of free TP calculated using the equation of Artursson and Karlsson was $8.219 \pm 1.145 \times 10^{-6} \mathrm{~cm} / \mathrm{s}$, which was consistent with the data from Gong et al. [17]. Obviously, The $P_{\text {app }}$ of MC-PLGA-TP-NP was much higher $\left(11.79 \pm 2.242 \times 10^{-6} \mathrm{~cm} / \mathrm{s}\right.$, Figure $\left.3(\mathrm{~b})\right)$, indicating that the designed nanoparticles could enhance the transcellular permeability of TP. According to the design of Transwell ${ }^{\mathrm{TM}}$ permeable supports, it is expected that drug permeated by passive absorption through transcellular or paracellular mechanisms can be observed in the basolateral compartment [27]. In this study, it is believed that the amount of TP determined in the basal chamber for both free TP and MC-PLGATP-NP can be attributed to the combination of passive transcellular and paracellular absorption mechanisms. In contrast, the higher TP amount in the basal chamber from MC-PLGATP-NP indicated that the multiple chemical coatings on the surface of nanoparticles can open intercellular junctions to accelerate the transcellular permeability of TP.
$\mathrm{A} \beta$ peptides, predominantly $\mathrm{A} \beta_{1-40}$ and $\mathrm{A} \beta_{1-42}$, are derived from the separation of the amyloid precursor protein [28]. Plenty of evidence have shown that an abnormal deposition of $\mathrm{A} \beta$ peptides in plaques of the brain tissue could cause the loss of neurons and synaptic lesions, which was considered the primary cause of the pathogenesis of $\mathrm{AD}$ [29]. At least partially, $A \beta$ neurotoxicity is induced by oxidative stress. Hence, therapeutic effort for attenuating the oxidative stress could be beneficial to $\mathrm{AD}$ treatment [19]. In the present study, we investigated the functions of TP and MC-PLGA-TP-NP in the cytotoxicity of $\mathrm{A} \beta_{1-42}$ on oxidative stress. Wang et al. reported that $\mathrm{A} \beta_{1-42}$ was cytotoxic to differentiated PC12 cells in a concentration-dependent manner. Herein, $5 \mu \mathrm{M} \mathrm{A} \beta_{1-42}$ as a final concentration was selected to induce the cytotoxicity for evaluating the protective effects of TP and MC-PLGA-TP-NP. As shown in Figure 4(a), $\mathrm{A} \beta_{1-42}$ obviously produced cytotoxicity without the protection of TP or MC-PLGA-TP-NP ( $0 \mathrm{ng} / \mathrm{ml}$ points), and only about $60 \%$ differentiated PC12 cells survived after incubation for $24 \mathrm{~h}$. With the increase of TP dosage in the TP group, the viability of $\mathrm{PC} 12$ cells increased until the concentration of $5 \mathrm{ng} / \mathrm{ml}$ of TP reached, which was consistent with the results from Xu's report [19]. However, the viability of PC12 cells suffered from a rapid decrease to about $50 \%$ when the concentration of TP was raised from $5 \mathrm{ng} / \mathrm{ml}$ to $10 \mathrm{ng} / \mathrm{ml}$, indicating that high dosage of TP could cause stronger toxicity and even reverse the protection effect. Notably, no obvious cytotoxicity was observed after the PC12 cells incubated with MC-PLGA-TP-NP in the whole range of prearranged concentrations. The reason may be that the sustained-release profile of MC-PLGA-TP-NP avoided the high dosage of TP interacting with cells. The results were acceptable and understandable because TP was a toxic chemical according to our 


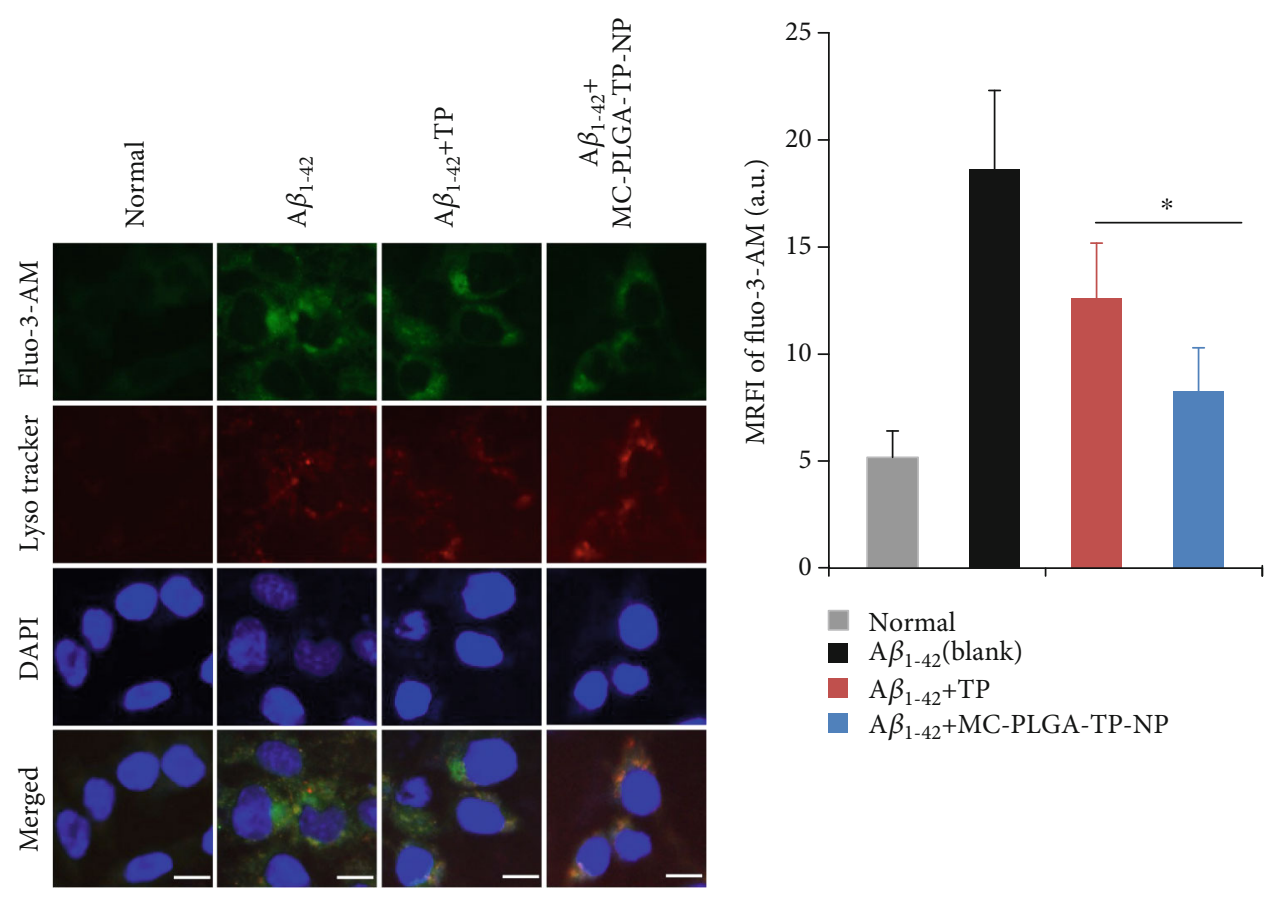

(a)

(b)

FIGURE 5: Fluorescence observation of calcium influx in the differentiated PC12 cells stained by different molecular probes. (a) The fluorescence images of the differentiated PC12 cells induced by A $\beta_{1-42}$ after the incubation with TP or MC-PLGA-TP-NP for 48 h. (b) Statistic data for the mean relative fluorescent intensity (MRFI) calculated by MetaMorph software. The experiment was repeated five times, and data were represented as the mean \pm standard deviation $(n=5)$. The scale bar represented $20 \mu \mathrm{m}$. ${ }^{*}$ Significant difference $(p<0.05$, versus TP).

previous study [6]. Thus, the development of new TP preparation to reduce its toxicity is a necessity.

It was reported that the protective functions of $\mathrm{TP}$ in $\mathrm{AD}$ may be via the pathway of oxidative stress. To evaluate the attenuation of the oxidative stress, the measurement of ROS generation closely associated with oxidative stress was carried out in this study. The results showed that the contents of ROS in the differentiated PC12 cells were increased significantly after the incubation with $\mathrm{A} \beta_{1-42}(p<0.05)$. Fortunately, the levels of intracellular ROS in differentiated PC12 cells for the treatment of both TP and MC-PLGA-TP-NP were significantly reduced compared to the blank group which was treated with $\mathrm{A} \beta_{1-42}$ only (Figure $4(\mathrm{~b})$ ), respectively, indicating that the treatment of the differentiated cells with TP or MC-PLGA-TP-NP can attenuate oxidative stress induced by $\mathrm{A} \beta_{1-42}$. There was no significant difference between the TP and MC-PLGA-TP-NP group $(p>0.05)$. Taken together, the designed MC-PLGA-TP-NP can exert an attenuation of the oxidative stress without any cytotoxicity to the differentiated PC12 cells.

$\mathrm{Ca}^{2+}$ is known as a second messenger in organisms, of which the disequilibrium is believed to be involved in the pathogenesis of $\mathrm{AD} . \mathrm{A} \beta$ can disrupt the calcium channels in cellular membrane, enhancing $\mathrm{Ca}^{2+}$ influx and then leading to the disequilibrium of calcium. Subsequently, the overload of intracellular $\mathrm{Ca}^{2+}$ is able to induce cytotoxicity and eventual cell death [30]. To further prove the neuroprotection of MC-PLGA-TP-NP, its effect on calcium influx of dif- ferentiated PC12 cells after the treatment of $\mathrm{A} \beta_{1-42}$ was evaluated. The resulting fluorescence images were shown in Figure 5, among which the green fluorescence corresponded to calcium deposition stained by Fluo-3-AM fluorescence and the red ones represented the lysosomes stained by LysoTracker Red in the PC12 cells, and the blue ones indicated the nuclei stained by DAPI (Figure 5(a)). It was evident that the coincubation of $\mathrm{A} \beta_{1-42}$ with the differentiated PC12 cells enhanced the calcium influx, and the mean relative fluorescent intensity (MRFI) of Fluo-3-AM fluorescence in the cells was approximately 3.6-fold higher than that in normal PC12cells (18.6 \pm 3.68 for cells treated with $\mathrm{A} \beta_{1-42}$ only vs. $5.17 \pm 1.24$ for normal cells) as shown in Figure 5(b). The increase in intracellular $\mathrm{Ca}^{2+}$ levels was remarkably inhibited by the treatment of TP or MC-PLGA-TP-NP (Figures 5(a) and 5(b)). Furthermore, MC-PLGA-TP-NP exhibited the better inhibition effect on calcium influx $(8.25 \pm 2.04$ vs. $12.62 \pm 2.56$ for $\mathrm{TP}, p<0.05)$. In comparison of other groups, the strongest red fluorescence was observed, corresponding to the endocytosis of MC-PLGA-TP-NP into the cells. Colocalization of both the red and the green stains showing orange-yellow color indicated that plenty of MCPLGA-TP-NP was transported into lysosome where lysosomal acidification drove $\mathrm{Ca}^{2+}$ influx [31]. In combination, the obtained data suggested that MC-PLGA-TP-NP may exert a better neuroprotective activity in the $\mathrm{AD}$ cellular model via following pharmacological mechanisms at least [1] inhibiting cytotoxicity and increasing the intracellular 
$\mathrm{Ca}^{2+}$ influx induced by $\mathrm{A} \beta$, [2] attenuating the oxidative stress in cells. However, whether MC-PLGA-TP-NP has an obvious therapeutic effect on $\mathrm{AD}$ models in vivo has not been studied. We are aware that our claims would require confirmation after in vivo animal tests, including biodistribution and therapy efficiency of MC-PLGA-TP-NP on the transgenic animal model of $\mathrm{AD}$.

\section{Conclusion}

We successfully prepared MC-PLGA-TP-NP showing sustained-release profile and better transcellular permeability to Caco- 2 cells in comparison of TP. In addition, MC-PLGATP-NP significantly reduced the cytotoxicity, attenuated the oxidative stress, and inhibited the increase of the intracellular $\mathrm{Ca}^{2+}$ influx in differentiated PC12 cells induced by $\mathrm{A} \beta_{1-42}$. Therefore, it can be concluded that MC-PLGA-TP-NP should be a more promising preparation of TP, which may exert a better neuroprotective activity in the $\mathrm{AD}$ cellular model.

\section{Data Availability}

The data used to support the findings of this study are available from the corresponding author upon request.

\section{Conflicts of Interest}

The authors declare that there is no conflict of interest.

\section{Acknowledgments}

This work was supported by the project funded by China Postdoctoral Science Foundation (No. 2018M641679) and project funded by the Key Research and Development Projects Science of Shanxi Province (201803D31165).

\section{References}

[1] Q. Wang, B. Xiao, S. Cui et al., “Triptolide treatment reduces Alzheimer's disease (AD)-like pathology through inhibition of BACE1 in a transgenic mouse model of AD," Disease Models \& Mechanisms, vol. 7, no. 12, pp. 1385-1395, 2014.

[2] R. Belfiore, A. Rodin, E. Ferreira et al., "Temporal and regional progression of Alzheimer's disease-like pathology in 3xTg-AD mice," Aging Cell, vol. 18, no. 1, article e12873, 2019.

[3] J. M. Li, Y. Zhang, L. Tang et al., "Effects of triptolide on hippocampal microglial cells and astrocytes in the APP/PS1 double transgenic mouse model of Alzheimer's disease," Neural Regeneration Research, vol. 11, no. 9, pp. 1492-1498, 2016.

[4] F. Regen, J. Hellmann-Regen, E. Costantini, and M. Reale, "Neuroinflammation and Alzheimer's disease: implications for microglial activation," Current Alzheimer Research, vol. 14, no. 11, pp. 1140-1148, 2017.

[5] B. J. Chen, "Triptolide, a novel immunosuppressive and antiinflammatory agent purified from a Chinese herb Tripterygium Wilfordii hook F," Leukemia \& Lymphoma, vol. 42, pp. 253-265, 2001.

[6] Z. X. Yuan, X. J. Wu, J. Mo, Y. L. Wang, C. Q. Xu, and L. Y. Lim, "Renal targeted delivery of triptolide by conjugation to the fragment peptide of human serum albumin," European
Journal of Pharmaceutics and Biopharmaceutics, vol. 94, pp. 363-371, 2015.

[7] Y. Zheng, W. J. Zhang, and X. M. Wang, "Triptolide with potential medicinal value for diseases of the central nervous system," CNS Neuroscience \& Therapeutics, vol. 19, pp. 7682, 2013.

[8] S. Cheng, K. J. LeBlanc, and L. Li, “Triptolide preserves cognitive function and reduces neuropathology in a mouse model of Alzheimer's se," PLoS One, vol. 9, no. 9, article e108845, 2014.

[9] A. R. Khan, M. Liu, M. W. Khan, and G. Zhai, "Progress in brain targeting drug delivery system by nasal route," Journal of Controlled Release, vol. 268, pp. 364-389, 2017.

[10] A. O. Elzoghby, W. S. Abo el-Fotoh, and N. A. Elgindy, "Casein-based formulations as promising controlled release drug delivery systems," Journal of Controlled Release, vol. 153, no. 3, pp. 206-216, 2011.

[11] F. Qi, J. Wu, H. Li, G. Ma, and G. Ma, "Recent Research and Development of PLGA/PLA Microspheres/Nanoparticles: A Review in Scientific and Industrial Aspects," Frontiers of Chemical Science and Engineering, vol. 13, no. 1, pp. 14-27, 2019.

[12] B. Wu, S. T. Lu, L. J. Zhang, R. X. Zhuo, H. B. Xu, and S. W. Huang, "Codelivery of doxorubicin and triptolide with reduction-sensitive lipid-polymer hybrid nanoparticles for in vitro and in vivo synergistic cancer treatment," International Journal of Nanomedicine, vol. 12, pp. 1853-1862, 2017.

[13] J. Bian, Z. Yuan, X. Chen, Y. Gao, C. Xu, and J. Shi, "Preparation of surface multiple-coated polylactide acid drug-loaded nanoparticles for intranasal delivery and evaluation on its brain-targeting efficiency," Drug Delivery, vol. 23, no. 1, pp. 269-276, 2016.

[14] Z. X. Yuan, L. Jia, L. Y. Lim et al., "Renal-targeted delivery of triptolide by entrapment in pegylated TRX-20-modified liposomes," International Journal of Nanomedicine, vol. 12, pp. 5673-5686, 2017.

[15] Z. H. Peng, J. J. Wang, P. Du, and Y. Chen, "Identification of in vivo and in vitro metabolites of triptolide by liquid chromatography-tandem mass spectrometry," Journal of Pharmaceutical and Biomedical Analysis, vol. 70, pp. 624-630, 2012.

[16] M. Rahbarian, E. Mortazavian, F. A. Dorkoosh, and M. Rafiee Tehrani, "Preparation, Evaluation and Optimization of Nanoparticles Composed of Thiolated Triethyl Chitosan: A Potential Approach for Buccal Delivery of Insulin," Journal of Drug Delivery Science \& Technology, vol. 44, pp. 254-263, 2018.

[17] X. Gong, Y. Chen, and Y. Wu, "Absorption and metabolism characteristics of triptolide as determined by a sensitive and reliable LC-MS/MS method," Molecules, vol. 20, no. 5, pp. 8928-8940, 2015.

[18] H. Wang, T. Jiang, W. Li, N. Gao, and T. Zhang, "Resveratrol attenuates oxidative damage through activating mitophagy in an in vitro model of Alzheimer's disease," Toxicology Letters, vol. 282, pp. 100-108, 2018.

[19] P. Xu, Z. Li, H. Wang, X. Zhang, and Z. Yang, "Triptolide inhibited cytotoxicity of differentiated PC12 cells induced by amyloid-Beta25-35 via the autophagy pathway," PLoS One, vol. 10, no. 11, article e0142719, 2015.

[20] M. Liu, J. Dong, Y. Yang, X. Yang, and H. Xu, "Characterization and release of triptolide-loaded poly (D, L-lactic acid) nanoparticles," European Polymer Journal, vol. 41, no. 2, pp. 375-382, 2005. 
[21] M. Xue, Y. Zhao, X. J. Li et al., "Comparison of toxicokinetic and tissue distribution of triptolide-loaded solid lipid nanoparticles vs free triptolide in rats," European Journal of Pharmaceutical Sciences, vol. 47, no. 4, pp. 713-717, 2012.

[22] J. Liu, H. Cheng, L. Han et al., "Synergistic combination therapy of lung cancer using paclitaxel- and triptolide-coloaded lipid\&ndash;polymer hybrid nanoparticles," Drug Design, Development and Therapy, vol. 12, pp. 3199-3209, 2018.

[23] L. Priano, D. Esposti, R. Esposti et al., "Solid lipid nanoparticles incorporating melatonin as new model for sustained oral and transdermal delivery systems," Journal of Nanoscience and Nanotechnology, vol. 7, no. 10, pp. 3596-3601, 2007.

[24] G. Rassu, E. Soddu, A. M. Posadino et al., "Nose-to-brain delivery of BACE1 siRNA loaded in solid lipid nanoparticles for Alzheimer's therapy," Colloids and Surfaces B: Biointerfaces, vol. 152, pp. 296-301, 2017.

[25] E. Muntimadugu, R. Dhommati, A. Jain, V. G. Challa, M. Shaheen, and W. Khan, "Intranasal delivery of nanoparticle encapsulated tarenflurbil: a potential brain targeting strategy for Alzheimer's disease," European Journal of Pharmaceutical Sciences, vol. 92, pp. 224-234, 2016.

[26] T. Musumeci, R. Pellitteri, M. Spatuzza, and G. Puglisi, "Noseto-brain delivery: evaluation of polymeric nanoparticles on olfactory ensheathing cells uptake," Journal of Pharmaceutical Sciences, vol. 103, no. 2, pp. 628-635, 2014.

[27] M. K. Shah, P. Madan, and S. Lin, "Elucidation of intestinal absorption mechanism of carvedilol-loaded solid lipid nanoparticles using Caco-2 cell line as an in-vitro model," Pharmaceutical Development and Technology, vol. 20, pp. 877-885, 2015.

[28] O. O. Olubiyi and B. Strodel, "Structures of the amyloid $\beta$-peptides $\mathrm{A} \beta 1-40$ and $\mathrm{A} \beta 1-42$ as influenced by $\mathrm{pH}$ and a D-peptide," The Journal of Physical Chemistry B, vol. 116, no. 10, pp. 3280-3291, 2012.

[29] E. Tamagno, M. Guglielmotto, D. Monteleone, G. Manassero, V. Vasciaveo, and M. Tabaton, "The unexpected role of A $\beta 1$ 42 monomers in the pathogenesis of Alzheimer's disease," Journal of Alzheimer's Disease, vol. 62, no. 3, pp. 1241-1245, 2018.

[30] X. Sun, L. Jin, and P. Ling, "Review of drugs for Alzheimer's disease," Drug discoveries \& therapeutics, vol. 6, pp. 285-290, 2012.

[31] X. Z. Zhong, Y. Yang, X. Sun, and X. P. Dong, "Methods for monitoring $\mathrm{Ca} 2+$ and ion channels in the lysosome," Cell Calcium, vol. 64, pp. 20-28, 2017. 